\title{
DESIGN, SYNTHESIS AND ANALYSIS OF ANTICANCER ACTIVITY OF NEW SAR-BASED S16020 DERIVATIVES
}

\author{
BEATA TYLIŃSKA ${ }^{*}$, RYSZARD JASZTOLD-HOWORKO 1 , \\ KARINA KOWALCZEWSKA ${ }^{1}$, KATARZYNA SZCZAURSKA-NOWAK ${ }^{2}$, \\ TOMASZ GĘBAROWSKI ${ }^{3}$ and JOANNA WIETRZYK ${ }^{2}$ \\ ${ }^{1}$ Department of Organic Chemistry, Faculty of Pharmacy, \\ Medical University of Wrocław, Poland \\ ${ }^{2}$ Institute of Immunology and Experimental Therapy, \\ Polish Academy of Sciences, Wrocław, Poland \\ ${ }^{3}$ Department of Basic Medical Sciences, Faculty of Pharmacy, \\ Medical University of Wrocław, Poland
}

\begin{abstract}
A novel set of S16020 derivatives was designed applying structure-activity relationships (SAR). The newly synthesized compounds were subjected to in vitro cytostatic activity screening against human kidney cancer (cell line A498), human lung cancer (cell line A549) and normal human dermal fibroblasts (cell line NHDF). Two $6 H$-pyrido[4,3- $b]$ carbazole derivatives exhibited stronger potency against both investigated cell lines than the reference compounds ellipticine and doxorubicin.
\end{abstract}

Keywords: olivacine, cytostatic, A498, A549, NHDF, S16020

Structure-activity relationships (SAR) is a method based on a simple and fundamental discovery that the biological or physicochemical activity of a chemical compound relates to its structure. Hence the simple assumption that similar molecules have similar activities and minor modifications (e.g. replacement of substituents, magnification of the molecule, ring condensation etc.) to the molecular structure of the active substance could yield compounds with even better activity or selectivity, and/or improved pharmacokinetic properties (1-3). Pyridocarbazole- based compounds, like ellipticine<smiles>Cc1c2ccnc(C(=O)NCCN(C)C)c2cc2c3cc(O)ccc3n(C)c12</smiles>

Figure 1. Chemical structure of 9-hydroxy-5,6-dimethyl- $N$-[2(dimethylamino)ethyl]-6H-pyrido[4,3- $b$ ]carbazole-1-carboxamide S16020 and structurally connected olivacine, have shown significant biological activity. One of the pyridocarbazole-based derivatives 9-hydroxy-5,6-dimethyl$\mathrm{N}$-[2-(dimethylamino)ethyl]-6H-pyrido[4,3-b]carbazole-1-carboxamide (S16020) (Fig. 1) has displayed impressive cytotoxic potency (4-5). The compound has shown marked antitumor activity against human (lung, breast, colon, and ovary) and murine (B16 melanoma, P388 leukemia, Lewis lung carcinoma, M5076 sarcoma) tumor models (6-12). Importantly, S 16020 has already been tested in a few clinical trials $(13,14)$ and confirms suggestions that the most active compounds act as DNA intercalators. One of the new series of $6 \mathrm{H}$-pyrido[4,3b]carbazole derivatives: 9-hydroxy-5,6-dimethyl- $N$ [2-(dimethylamino)ethyl]-6H-pyrido[4,3-b]carbazole-1-carboxamide $\mathbf{1}$ is an intercalating compound exhibiting a high DNA binding affinity and inhibition of topoisomerase II, which is responsible for its pharmacological activity $(8,15)$. Using literature data and based on our results (16-20) a new set of new pyrido[4,3- $b$ carbazole derivatives were designed and synthesized. The novel compounds

* Corresponding author: e-mail: beata.tylinska@umed.wroc.pl 


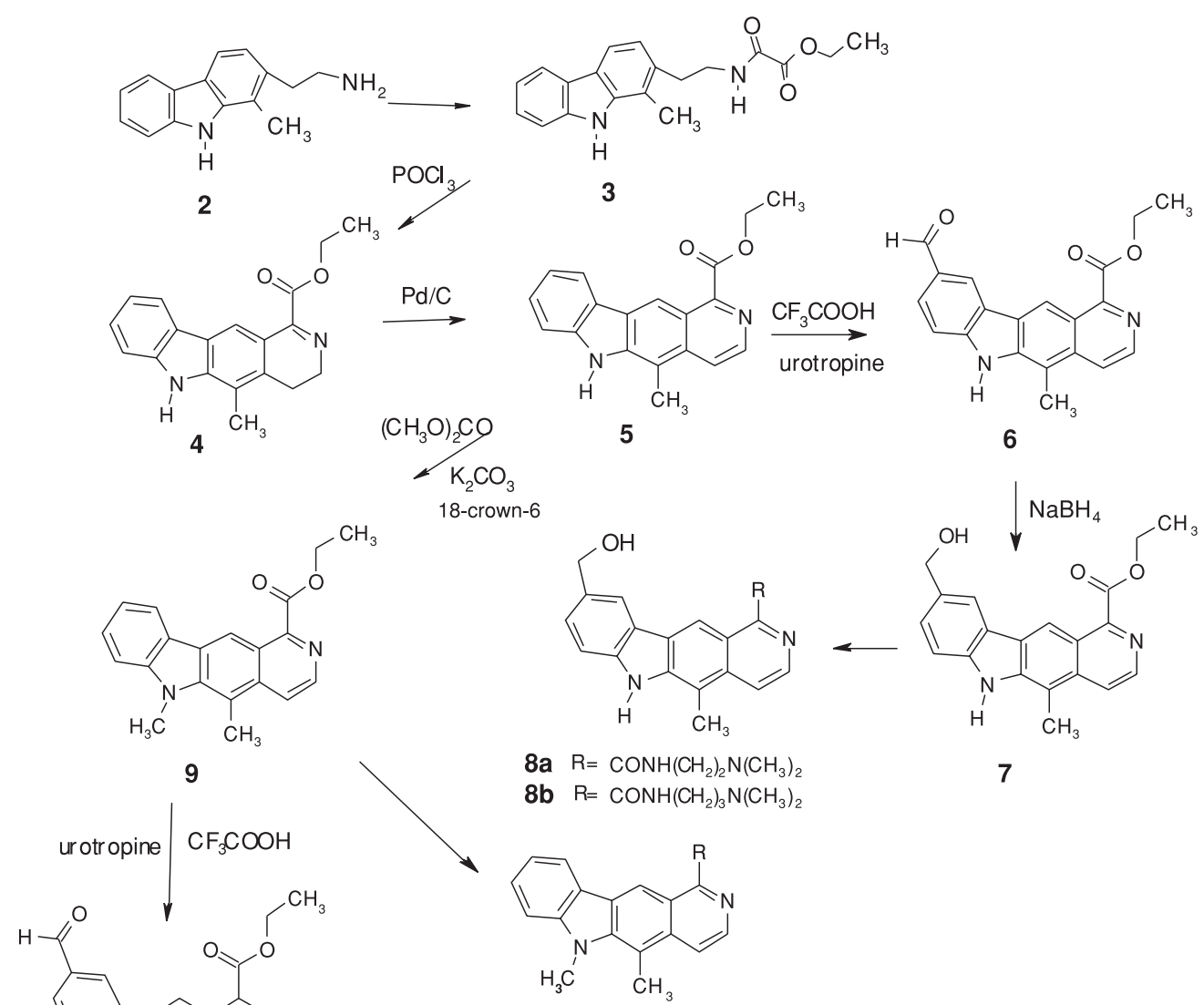

$10 \mathbf{a} \mathrm{R}=\mathrm{CONH}\left(\mathrm{CH}_{2}\right)_{2} \mathrm{~N}\left(\mathrm{CH}_{3}\right)_{2}$ 10b $\mathrm{R}=\mathrm{CONHC}\left(\mathrm{CH}_{3}\right)_{2} \mathrm{CH}_{2} \mathrm{OH}$

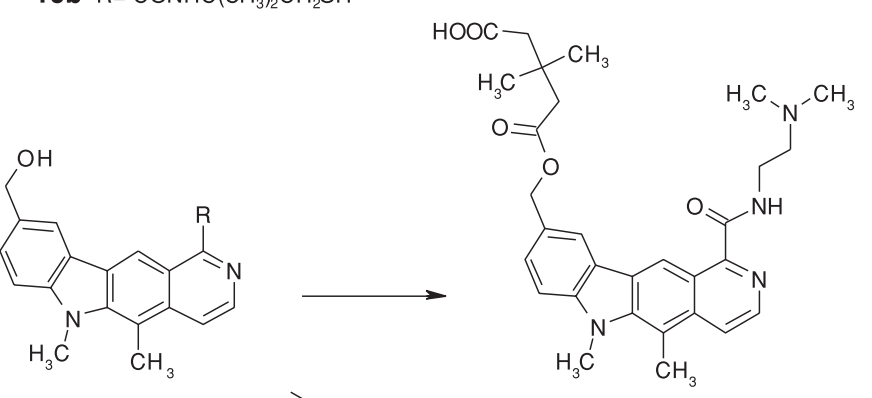

12
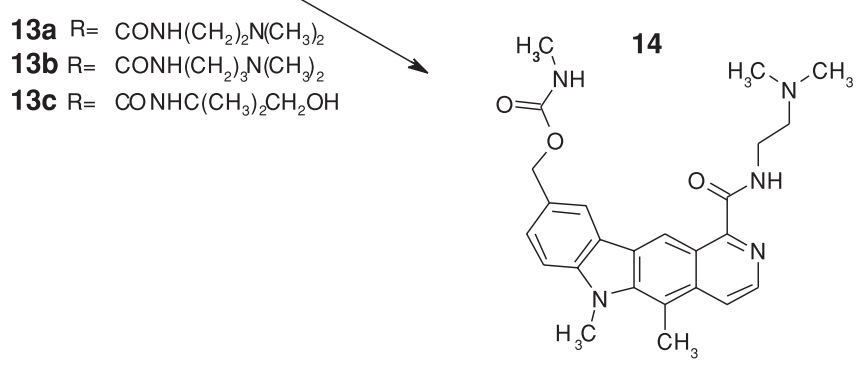

15

Scheme 1. Synthesis of S16020 derivatives 
Table 1. Cell growth inhibition of A498 (kidney cancer), A549 (non-small-cell lung cancer) and NHDF (Normal Human Dermal Fibroblasts) cells. $\mathrm{IC}_{50}$ values $[\mu \mathrm{M}] \pm \mathrm{SD}$ of compounds $8 \mathrm{a}-15$ compared to ellipticine.

\begin{tabular}{|c|c|c|c|}
\hline $\begin{array}{c}\text { Compound } \\
\text { No }\end{array}$ & $\begin{array}{c}\mathrm{A} 498 \\
\mathrm{IC}_{50}[\mu \mathrm{M}] \pm S D\end{array}$ & $\begin{array}{c}\mathrm{A} 549 \\
\mathrm{IC}_{50}[\mu \mathrm{M}] \pm S D\end{array}$ & $\begin{array}{c}\mathrm{NHDF} \\
\mathrm{IC}_{50}[\mu \mathrm{M}] \pm S D\end{array}$ \\
\hline $8 \mathrm{a}$ & $5.03 \pm 3.14$ & $1.50 \pm 0.29$ & $6.77 \pm 0.68$ \\
\hline $8 \mathrm{~b}$ & $9.17 \pm 1.92$ & $10.4 \pm 2.15$ & $12.1 \pm 1.44$ \\
\hline $10 \mathrm{a}$ & $3.24 \pm 0.11$ & $2.69 \pm 1.16$ & $12.8 \pm 0.19$ \\
\hline $10 \mathrm{~b}$ & $13.3 \pm 2.65$ & $19.58 \pm 1.57$ & $33.07 \pm 7.42$ \\
\hline $13 \mathrm{a}$ & $0.78 \pm 0.13$ & $0.65 \pm 0.3$ & $7.33 \pm 1.11$ \\
\hline $13 \mathrm{~b}$ & $5.42 \pm 2.30$ & $4.46 \pm 0.44$ & $14.1 \pm 1.06$ \\
\hline $13 \mathrm{c}$ & $1.30 \pm 0.33$ & $7.15 \pm 3.83$ & $24.5 \pm 4.06$ \\
\hline 14 & $2.45 \pm 1.03$ & $5.70 \pm 4.50$ & $8.6 \pm 2.08$ \\
\hline 15 & $0.84 \pm 0.11$ & $1.25 \pm 0.29$ & $8.93 \pm 0.73$ \\
\hline Ellipticine & $1.74 \pm 0.04$ & $0.85 \pm 0.04$ & $6.32 \pm 0.55$ \\
\hline Doxorubicin & $0.70 \pm 0.17$ & $0.99 \pm 0.32$ & 0.71 \\
\hline
\end{tabular}

were synthesized according to Scheme 1. Cytostatic activity in vitro of all newly synthesized compounds was examined using kidney cancer (A498), lung cancer (A549) and normal (healthy) human fibroblasts (NHDF) cell lines. Some of the investigated compounds showed strong cytostatic properties with a broad spectrum of antitumor activity when compared to ellipticine. The activity of two of novel compounds is comparable to that exhibited by the reference compound of doxorubicin. All the tested compounds were also characterized by low toxicity to normal cells (Table 1).

Compound 15 (Polish patent- PL-218501) outperformed ellipticine antiproliferative activity on the A498 tumor cell line.

\section{MATERIALS AND METHODS}

\section{Instrumentation}

Melting points were determined using a Köfler apparatus and were uncorrected; ${ }^{1} \mathrm{H}$ NMR spectra were recorded on a Bruker 300 at $300.14 \mathrm{MHz}$, using TMS as an internal standard. Column chromatography was carried out on silica gel (Merck Kieselgel 100). All of the newly obtained compounds were analyzed for $\mathrm{C}, \mathrm{H}$, and $\mathrm{N}$, and the analytical results were within $\pm 0.4 \%$ of theoretical values.

\section{Synthetic chemistry}

The starting compound 2-(1-methyl-9H-carbazol-2-yl)ethylamine $\mathbf{2}$ was prepared according to a previously described procedure (24). Compound 2 was reacted with diethyl oxalate and after cycliza- tion of the resulting amide 3 gave 1-ethoxycarbonyl5-methyl-3,4-dihydro-6H-pyrido[4,3- $b]$ carbazole 4, which was further aromatized to obtain derivative $\mathbf{5}$. Compound 6 was obtained via heating of 1-ethoxycarbonyl-5-methyl-6H-pyrido[4,3- $b]$ carbazole $\mathbf{5}$ with trifluoroacetic acid (TFA) and urotropine. The 1-ethoxycarbonyl-9-formyl-5-methyl-6H-pyrido[4,3-b]carbazole 6 was reduced to compound 7 . Derivatives 8a-b were prepared in reaction of 7 with 2-dimethyl-aminoethylamine or 3-dimethyl-aminopropylamine respectively. Methylation of $\mathbf{5}$ at N-6 position resulted in derivative 9. Amides 10a-b were prepared reacting 9 with 2-dimethyl-aminoethylamine or 2-amino-2-methyl-1-propanol respectively. Compounds 13a-c were synthesized starting from 9 and using a procedure similar to one described for compounds 8a-b. Derivative 13a was boiled in pyridine with 3,3-dimethylglutaric anhydride giving 14. The most active compound 15 (see Biological Results) was obtained through reaction of 13a with methyl isocyanate (Scheme 1).

\section{N-[2-(1-methyl-9H-carbazole-2-yl)ethyl]-1-ethoxy- carbonylamid (3)}

2-(2-aminoethyl)-1-methylcarbazol 2 (1.12 g, $5 \mathrm{mmol})$ was dissolved in diethyl oxalate $(10 \mathrm{~mL})$. The mixture was heated to $120^{\circ} \mathrm{C}$ and maintained for $2 \mathrm{~h}$. After evaporation to dryness, the solid was purified using column chromatography and silica gel column, eluting with methylene chloride. Yield: $83 \%$, mp: $183^{\circ} \mathrm{C}$. Analysis: calcd. for $\mathrm{C}_{19} \mathrm{H}_{20} \mathrm{~N}_{2} \mathrm{O}_{3}$ : C, 70.35; H, 6.21; N, 8.64\%; found: C, 70.10; H, 6.34; N, 8.42\%. ${ }^{1} \mathrm{H}$ NMR (DMSO-d $\left.\mathrm{d}_{6}\right) \delta: 1.25(\mathrm{~m}$, $\left.3 \mathrm{H}, \mathrm{CH}_{2} \mathrm{CH}_{3}\right), 2.52$ (s, 3H,1- $\left.\mathrm{CH}_{3}\right), 2.93$ (m, 2H, $\beta$ - 
$\left.\mathrm{CH}_{2}\right), 3.25\left(\mathrm{~m}, 2 \mathrm{H}, \alpha-\mathrm{CH}_{2}\right), 4.21\left(\mathrm{~m}, 2 \mathrm{H}, \mathrm{CH}_{2} \mathrm{CH}_{3}\right)$, $6.95\left(\mathrm{~d}, J_{3-4}=7.9 \mathrm{~Hz}, 1 \mathrm{H}, 3-\mathrm{H}\right), 7.10\left(\mathrm{~d}, J_{7-5}=7.6\right.$ $\mathrm{Hz}, 1 \mathrm{H}, 7-\mathrm{H}), 7.32\left(\mathrm{~d}, J_{6-8}=8.0 \mathrm{~Hz}, 1 \mathrm{H}, 6-\mathrm{H}\right), 7.46$ $\left(\mathrm{d}, J_{8-6}=8.0 \mathrm{~Hz}, 1 \mathrm{H}, 8-\mathrm{H}\right), 7.83\left(\mathrm{~d}, J_{4-3}=7.9 \mathrm{~Hz}, 1 \mathrm{H}\right.$, 4-H), $8.01\left(\mathrm{~d}, J_{5-7}=7.6 \mathrm{~Hz}, 1 \mathrm{H}, 5-\mathrm{H}\right), 9.05(\mathrm{t}, J=5.2$ $\mathrm{Hz}, 1 \mathrm{H},-\mathrm{NHCO}), 11.05$ (s, 1H, 9-NH).

\section{1-ethoxycarbonyl-5-methyl-3,4-dihydro-6H-pyri- do[4,3-b]carbazole (4)}

The compound $3(0.97 \mathrm{~g}, 3 \mathrm{mmol})$ was dissolved in boiling toluene $(110 \mathrm{~mL})$ and then treated dropwise with phosphorous oxychloride $(10 \mathrm{~mL})$. The reflux was continued for $12 \mathrm{~h}$ following with evaporation under reduced pressure that resulted in a residue that was solubilized in water $(100 \mathrm{~mL})$, alkalized to $\mathrm{pH}$ 9-10 with sodium hydrogen carbonate, and extracted with methylene chloride. The extract was dried over magnesium sulfate. After evaporation of the solvent, the solid was purified using column chromatography and silica gel, eluting with methylene chloride: methanol, $98: 2$ v/v. Yield: $43 \%$; mp: $185-186^{\circ} \mathrm{C}$. Analysis: calcd. for $\mathrm{C}_{19} \mathrm{H}_{18} \mathrm{~N}_{2} \mathrm{O}_{2}$ : C, 74.49; H, 5.92; N, 9.14\%; found: $\mathrm{C}$, $74.34 ; \mathrm{H}, 6.11 ; \mathrm{N}, 8.92 \%$. ${ }^{1} \mathrm{H}$ NMR $\left(\mathrm{DMSO}_{-} \mathrm{d}_{6}\right) \delta$ : $1.33\left(\mathrm{t}, 3 \mathrm{H},-\mathrm{CH}_{2} \mathrm{CH}_{3}\right), 2.52\left(\mathrm{~s}, 3 \mathrm{H}, 5-\mathrm{CH}_{3}\right), 2.83(\mathrm{~m}$, $\left.2 \mathrm{H}, 3-\mathrm{CH}_{2}\right), 3.72\left(\mathrm{~m}, 2 \mathrm{H}, 4-\mathrm{CH}_{2}\right), 4.38(\mathrm{q}, 2 \mathrm{H},-$ $\left.\mathrm{CH}_{2} \mathrm{CH}_{3}\right), 7.17\left(\mathrm{t}, J_{8-10}=7.5 \mathrm{~Hz}, 1 \mathrm{H}, 8-\mathrm{H}\right), 7.39\left(\mathrm{t}, J_{9-7}\right.$ $=8.0 \mathrm{~Hz}, 1 \mathrm{H}, 9-\mathrm{H}), 7.51\left(\mathrm{~d}, J_{7-9}=8.0 \mathrm{~Hz}, 1 \mathrm{H}, 7-\mathrm{H}\right)$, $8.02(\mathrm{~s}, 1 \mathrm{H}, 11-\mathrm{H}), 8.12\left(\mathrm{~d}, J_{10-8}=7.7 \mathrm{~Hz}, 1 \mathrm{H}, 10-\mathrm{H}\right)$, 11.50 (s, 1H, 6-NH).

\section{1-ethoxycarbonyl-5-methyl-6H-pyrido[4,3-b]car- bazole (5)}

A mixture of $4(0.92 \mathrm{~g}, 3 \mathrm{mmol}), 10 \%$ palladium on charcoal $(0.10 \mathrm{~g})$ and decalin $(50 \mathrm{~mL})$ was stirred at boiling temperature for $30 \mathrm{~min}$. The catalyst was filtered away. The yellow precipitate was filtered and purified using column chromatography and silica gel, eluting with methylene chloride: methanol, $99: 1$ v/v. Yield: $55 \%$; mp: $205^{\circ} \mathrm{C}$. Analysis: calcd. for $\mathrm{C}_{19} \mathrm{H}_{16} \mathrm{~N}_{2} \mathrm{O}_{2}$ : C, 74.98; H, 5.30; N, 9.20\%; found: C, 74.69; H, 5.45; N, 9.01\%. ${ }^{1} \mathrm{H}$ NMR $\left(\right.$ DMSO-d $\left._{6}\right) \delta$ : $1.42\left(\mathrm{t}, 3 \mathrm{H},-\mathrm{CH}_{2} \mathrm{CH}_{3}\right), 2.87$ (s, 3H, 5- $\left.\mathrm{CH}_{3}\right), 4.55$ (q, $\left.2 \mathrm{H},-\mathrm{CH}_{2} \mathrm{CH}_{3}\right), 7.26(\mathrm{~m}, 1 \mathrm{H}, 8-\mathrm{H}), 7.55(\mathrm{~m}, 2 \mathrm{H}, 7-\mathrm{H}$, 9-H), $8.15\left(\mathrm{~d}, J_{4-3}=6.0 \mathrm{~Hz}, 1 \mathrm{H}, 4-\mathrm{H}\right), 8.34\left(\mathrm{~d}, J_{10-8}=\right.$ $7.7 \mathrm{~Hz}, 1 \mathrm{H}, 10-\mathrm{H}), 8.45$ (d, $\left.J_{3-4}=5.9 \mathrm{~Hz}, 1 \mathrm{H}, 3-\mathrm{H}\right)$, 8.97 (s, 1H, 11-H), 11.55 (s, 1H, 6-NH).

1-ethoxycarbonyl-9-formyl-5-methyl-6H-pyrido[4,3-b]carbazole (6)

1-ethoxycarbonyl-9-formyl-5,6-dimethyl-6H-pyrido[4,3-b]carbazole (11)

A mixture of $0.5 \mathrm{mmol}$ compound 5 (or compound 9), $20 \mathrm{~mL}$ trifluoroacetic acid and $0.71 \mathrm{~g}(0.5$ mmol) hexamethylenetetramine was stirred at boiling temperature for $20 \mathrm{~min}$. After evaporation under reduced pressure the resulting compound was solubilized in water (100 mL), alkalized to $\mathrm{pH} 9-10$ with sodium hydrogen carbonate, and extracted with methylene chloride. The extract was dried over magnesium sulfate. After evaporation of the solvent, the solid was purified using column chromatography and silica gel, eluting with methylene chloride: methanol, $99: 1 \mathrm{v} / \mathrm{v}$.

\section{1-ethoxycarbonyl-9-formyl-5-methyl-6H-pyri- do[4,3-b]carbazole (6)}

Yield: $36 \%$; mp: $194-195^{\circ} \mathrm{C}$. Analysis: calcd. for $\mathrm{C}_{20} \mathrm{H}_{16} \mathrm{~N}_{2} \mathrm{O}_{3}$ : C, 72.28; H, 4.85; N, 8.43\%; found: C, 72.10; H, 5.04; N, 8.20\%. ${ }^{1} \mathrm{H}$ NMR $\left(\right.$ DMSO-d $\left._{6}\right) \delta$ : 1.42 (t, 3H, $-\mathrm{CH}_{2} \mathrm{CH}_{3}$ ), 2.87 (s, 3H, 5- $\mathrm{CH}_{3}$ ), 4.57 (q, $\left.2 \mathrm{H},-\mathrm{CH}_{2} \mathrm{CH}_{3}\right), 7.66\left(\mathrm{~d}, J_{8-7}=8.3,1 \mathrm{H}, 8-\mathrm{H}\right), 8.07(\mathrm{~d}$, $\left.J_{7-8}=8.4 \mathrm{~Hz}, 1 \mathrm{H}, 7-\mathrm{H},\right), 8.18\left(\mathrm{~d}, J_{4-3}=6.0 \mathrm{~Hz}, 1 \mathrm{H}, 4-\right.$ $\mathrm{H}), 8.48\left(\mathrm{~d}, J_{3-4}=6.0 \mathrm{~Hz}, 1 \mathrm{H}, 3-\mathrm{H}\right), 8.93(\mathrm{~s}, 1 \mathrm{H}, 10-$ H), 9.11 (s, 1H, 11-H), 10.07 (s, 1H, -CHO), 12.08 (s, 1H, 6-NH).

\section{1-ethoxycarbonyl-9-formyl-5,6-dimethyl-6H-pyri- do[4,3-b]carbazole (11)}

Yield: $81 \%$; mp: $240^{\circ} \mathrm{C}$. Analysis: calcd. for $\mathrm{C}_{21} \mathrm{H}_{18} \mathrm{~N}_{2} \mathrm{O}_{3}$ : C, 72.82; H, 5.24; N, 8.09\%; found: $\mathrm{C}$, 72.58; H, 5.37; N, 7.82\%. ${ }^{1} \mathrm{H}$ NMR $\left(\right.$ DMSO-d $\left._{6}\right) \delta$ : 1.42 (t, $\left.3 \mathrm{H},-\mathrm{CH}_{2} \mathrm{CH}_{3}\right), 3.11\left(\mathrm{~s}, 3 \mathrm{H}, 5-\mathrm{CH}_{3}\right), 4.24$ (s, $\left.3 \mathrm{H}, 6-\mathrm{CH}_{3}\right), 4.56\left(\mathrm{q}, 2 \mathrm{H},-\mathrm{CH}_{2} \mathrm{CH}_{3}\right), 7.80\left(\mathrm{~d}, J_{8-7}=\right.$ $8.4,1 \mathrm{H}, 8-\mathrm{H}), 8.11\left(\mathrm{~d}, J_{7-8}=8.4 \mathrm{~Hz}, 1 \mathrm{H}, 7-\mathrm{H},\right), 8.26$ $\left(\mathrm{d}, J_{4-3}=6.1 \mathrm{~Hz}, 1 \mathrm{H}, 4-\mathrm{H}\right), 8.50\left(\mathrm{~d}, J_{3-4}=6.1 \mathrm{~Hz}, 1 \mathrm{H}\right.$, $3-\mathrm{H}), 8.93$ (s, 1H, 10-H), 9.11 (s, 1H, 11-H), 10.08 (s, $1 \mathrm{H},-\mathrm{CHO})$.

1-ethoxycarbonyl-9-hydroxymethyl-5-methyl-6Hpyrido[4,3-b]carbazole (7)

1-ethoxycarbonyl-9-hydroxymethyl-5,6-dimethyl6H-pyrido[4, 3-b]carbazole (12)

The compound $\mathbf{6} 0.5 \mathrm{mmol}$ or compound $\mathbf{1 2}$ $0.5 \mathrm{mmol}, 90 \mathrm{~mL}$ THF and $0.20 \mathrm{~g}(5 \mathrm{mmol})$ sodium borohydride were stirred at room temperature for 2 h. After evaporation under reduced pressure the resulting compound was solubilized in water (100 $\mathrm{mL}$ ), acidified to $\mathrm{pH}$ 4-5 with hydrochloric acid, alkalized to $\mathrm{pH}$ 9-10 with sodium hydrogen carbonate, and extracted with methylene chloride. The extract was dried over magnesium sulfate. After evaporation of the solvent, the solid was purified using column chromatography and silica gel, eluting with methylene chloride: methanol, $98: 2 \mathrm{v} / \mathrm{v}$.

1-ethoxycarbonyl-9-hydroxymethyl-5-methyl-6Hpyrido[4,3-b]carbazole (7) 
Yield: $66 \%$; mp: $243^{\circ} \mathrm{C}$. Analysis: calcd. for $\mathrm{C}_{20} \mathrm{H}_{18} \mathrm{~N}_{2} \mathrm{O}_{3}$ : C, 71.84; H, 5.43; N, 8.38\%; found: $\mathrm{C}$, 71.66; H, 5.63; N, 8.11\%. ${ }^{1} \mathrm{H}$ NMR $\left(\right.$ DMSO-d $\left._{6}\right) \delta$ : $1.43\left(\mathrm{t}, 3 \mathrm{H},-\mathrm{CH}_{2} \mathrm{CH}_{3}\right), 2.87$ (s, 3H, 5- $\left.\mathrm{CH}_{3}\right), 4.56$ (q, $\left.2 \mathrm{H},-\mathrm{CH}_{2} \mathrm{CH}_{3}\right), 4.67$ (s, $\left.2 \mathrm{H},-\mathrm{CH}_{2} \mathrm{OH}\right), 5.27$ (s, $1 \mathrm{H}$, $\mathrm{OH}), 7.53(\mathrm{~m}, 2 \mathrm{H}, 8-\mathrm{H}, 7-\mathrm{H}), 8.15\left(\mathrm{~d}, J_{4-3}=6.0 \mathrm{~Hz}\right.$, $1 \mathrm{H}, 4-\mathrm{H}), 8.26(\mathrm{~s}, 1 \mathrm{H}, 10-\mathrm{H}), 8.45\left(\mathrm{~d}, J_{3-4}=6.0 \mathrm{~Hz}\right.$, $1 \mathrm{H}, 3-\mathrm{H}), 8.96(\mathrm{~s}, 1 \mathrm{H}, 11-\mathrm{H}), 11.60(\mathrm{~s}, 1 \mathrm{H}, 6-\mathrm{NH})$.

1-ethoxycarbonyl-9-hydroxymethyl-5,6-dimethyl6H-pyrido[4, 3-b]carbazole (12)

Yield: 58\%; mp: $196-197^{\circ} \mathrm{C}$. Analysis: calcd. for $\mathrm{C}_{21} \mathrm{H}_{20} \mathrm{~N}_{2} \mathrm{O}_{3}$ : C, 72.40; H, 5.79; N, 8.04\%, found: C, 72.27; H, 5.98; N, 7.83\%. ${ }^{1} \mathrm{H}$ NMR $\left(\right.$ DMSO-d $\left._{6}\right) \delta$ : 1.39 (t, $\left.3 \mathrm{H},-\mathrm{CH}_{2} \mathrm{CH}_{3}\right), 3.08\left(\mathrm{~s}, 3 \mathrm{H}, 5-\mathrm{CH}_{3}\right), 4.15$ (s, $\left.3 \mathrm{H}, 6-\mathrm{CH}_{3}\right), 4.55$ (q, $\left.2 \mathrm{H},-\mathrm{CH}_{2} \mathrm{CH}_{3}\right), 4.67$ (s, $2 \mathrm{H}$, $\left.\mathrm{CH}_{2} \mathrm{OH}\right), 5.27$ (s, 1H, -OH), 7.56 (m, 2H, 8-H,7-H), $8.20\left(\mathrm{~d}, J_{4-3}=6.0 \mathrm{~Hz}, 1 \mathrm{H}, 4-\mathrm{H}\right), 8.26(\mathrm{~s}, 1 \mathrm{H}, 10-\mathrm{H})$, $8.44\left(\mathrm{~d}, J_{3-4}=6.0 \mathrm{~Hz}, 1 \mathrm{H}, 3-\mathrm{H}\right), 8.95(\mathrm{~s}, 1 \mathrm{H}, 11-\mathrm{H})$.

\section{The synthesis of amides $8 a-b, 10 a-b, 13 a-c$}

A mixture of ( $0.5 \mathrm{mmol}) 6 \mathrm{H}$-pyrido[4,3- $b$ ]carbazole derivative 7 (or 9 or 12) and $10 \mathrm{~mL}$ corresponding amine was heated at reflux under nitrogen atmosphere. The reaction was performed until the disappearance of the starting material (TLC monitoring). After evaporation of the solvent, the solid was purified using column chromatography and silica gel, eluting with methylene chloride: methanol, $95: 5 \mathrm{v} / \mathrm{v}$.

9-hydroxymethyl-5-methyl-1-[N,N-(dimethylamino)ethyl]carbamoyl-6H-pyrido[4,3-b]carbazole (8a)

Yield: $57 \%$; mp: $248-249^{\circ} \mathrm{C}$. Analysis: calcd. for: $\mathrm{C}_{22} \mathrm{H}_{24} \mathrm{~N}_{4} \mathrm{O}_{2}: \mathrm{C}, 70.19 ; \mathrm{H}, 6.43 ; \mathrm{N}, 14.88 \%$, found: $\mathrm{C}, 69.99 ; \mathrm{H}, 6.56 ; \mathrm{N}, 14.73 \% .{ }^{1} \mathrm{H}$ NMR $\left(\mathrm{DMSO}_{6}\right) \delta: 2.24\left(\mathrm{~s}, 6 \mathrm{H},-\mathrm{N}\left(\mathrm{CH}_{3}\right)_{2}\right), 2.52(\mathrm{~m}, 2 \mathrm{H}$, $\left.\beta-\mathrm{CH}_{2}\right), 2.84$ (s, 3H, 5- $\left.\mathrm{CH}_{3}\right), 3.50$ (q, 2H, $\alpha-\mathrm{CH}_{2}$ ), $4.66\left(\mathrm{~s}, 2 \mathrm{H},-\mathrm{CH}_{2} \mathrm{OH}\right), 5.23(\mathrm{~s}, 1 \mathrm{H},-\mathrm{OH}), 7.49(\mathrm{~m}$, $2 \mathrm{H}, 8-\mathrm{H}, 7-\mathrm{H}), 8.11\left(\mathrm{~d}, J_{4-3}=6.0 \mathrm{~Hz}, 1 \mathrm{H}, 4-\mathrm{H}\right), 8.18$ (s, 1H, 10-H), $8.42\left(\mathrm{~d}, J_{3-4}=6.0 \mathrm{~Hz}, 1 \mathrm{H}, 3-\mathrm{H}\right), 8.90$ $\left(\mathrm{t}, 1 \mathrm{H},-\mathrm{CONHCH}_{2}\right), 9.66(\mathrm{~s}, 1 \mathrm{H}, 11-\mathrm{H}), 11.41$ (s, $1 \mathrm{H}, 6-\mathrm{NH})$.

9-hydroxymethyl-5-methyl-1-[N,N-(dimethylamino)propyl]carbamoyl-6H-pyrido[4,3-b]carbazole $(8 b)$

Yield: $51 \%$; mp: $244^{\circ} \mathrm{C}$. Analysis: calcd. for $\mathrm{C}_{23} \mathrm{H}_{26} \mathrm{~N}_{4} \mathrm{O}_{2}$ : C, 70.75; H, 6.71; N, $14.35 \%$; found: $\mathrm{C}$, 70.57; H, 6.92; N, 14.13\%. ${ }^{1} \mathrm{H}$ NMR $\left(\right.$ DMSO-d $\left._{6}\right) \delta$ : $1.74\left(\mathrm{~m}, 2 \mathrm{H}, \beta-\mathrm{CH}_{2}\right), 2.15\left(\mathrm{~s}, 6 \mathrm{H},-\mathrm{N}\left(\mathrm{CH}_{3}\right)_{2}\right), 2.32$ (t, $\left.2 \mathrm{H}, \gamma-\mathrm{CH}_{2}\right), 2.82\left(\mathrm{~s}, 3 \mathrm{H}, 5-\mathrm{CH}_{3}\right), 3.43(\mathrm{~m}, 2 \mathrm{H}, \alpha-$ $\left.\mathrm{CH}_{2}\right), 4.66\left(\mathrm{~s}, 2 \mathrm{H},-\mathrm{CH}_{2} \mathrm{OH}\right), 5.26(\mathrm{~s}, 1 \mathrm{H},-\mathrm{OH}), 7.50$ (m, 2H, 8-H, 7-H), 8.09 (d, $\left.J_{4-3}=6.0 \mathrm{~Hz}, 1 \mathrm{H}, 4-\mathrm{H}\right)$, $8.17(\mathrm{~s}, 1 \mathrm{H}, 10-\mathrm{H}), 8.41\left(\mathrm{~d}, J_{3-4}=6.0 \mathrm{~Hz}, 1 \mathrm{H}, 3-\mathrm{H}\right)$, $8.90\left(\mathrm{t}, J \mathrm{NH}-\mathrm{CH}_{2}=5.8 \mathrm{~Hz}, 1 \mathrm{H},-\mathrm{CONHCH}_{2}\right), 9.50$ (s, 1H, 11-H), 11.59 (s, 1H, 6-NH).

\section{5,6-dimethyl-1-[N,N-(dimethylamino)ethyl]car- bamoyl-6H-pyrido[4,3-b]carbazole (10a)}

Yield: $44 \%$; mp: $171-172^{\circ} \mathrm{C}$. Analysis: calcd. for $\mathrm{C}_{22} \mathrm{H}_{24} \mathrm{~N}_{4} \mathrm{O}$ : C, 73.31; H, 6.71; N, 15.54\%; found: C, 73.07; H, 6.84; N, 15.29\%.' ${ }^{1} \mathrm{H}$ NMR (DMSO-d $\left.\mathrm{d}_{6}\right)$ $\delta: 2.24\left(\mathrm{~s}, 6 \mathrm{H},-\mathrm{N}\left(\mathrm{CH}_{3}\right)_{2}\right), 2.52\left(\mathrm{~m}, 2 \mathrm{H}, \beta-\mathrm{CH}_{2}\right), 3.05$ (s, 3H, 5- $\mathrm{CH}_{3}$ ), 3.49 (m, 2H, $\left.\alpha-\mathrm{CH}_{2}\right), 4.12$ (s, 3H, 6$\left.\mathrm{CH}_{3}\right), 7.27(\mathrm{~m}, 1 \mathrm{H}, 8-\mathrm{H}), 7.58(\mathrm{~m}, 2 \mathrm{H}, 9-\mathrm{H}, 7-\mathrm{H})$, $8.15\left(\mathrm{~d}, J_{4-3}=6.1 \mathrm{~Hz}, 1 \mathrm{H}, 4-\mathrm{H}\right), 8.20\left(\mathrm{~d}, J_{10-8}=7.6\right.$ $\mathrm{Hz}, 1 \mathrm{H}, 10-\mathrm{H}), 8.41\left(\mathrm{~d}, J_{3-4}=6.1 \mathrm{~Hz}, 1 \mathrm{H}, 3-\mathrm{H}\right), 8.76$ (t, $\left.1 \mathrm{H},-\mathrm{CONHCH}_{2}\right), 9.66$ (s, 1H, 11-H).

\section{5,6-dimethyl-1-(2-hydroxy-1,1-dimethylethyl)car-} bamoyl-6H-pyrido[4,3-b]carbazole (10b)

Yield: $34 \%$; mp: $213^{\circ} \mathrm{C}$. Analysis: calcd. for $\mathrm{C}_{22} \mathrm{H}_{23} \mathrm{~N}_{3} \mathrm{O}_{2}$ : C, 73.11; H, 6.41; N, $11.63 \%$; found: $\mathrm{C}$, 72.92; H, 6.56; N, 11.43\%. ${ }^{1} \mathrm{H}$ NMR (DMSO-d $\left.{ }_{6}\right) \delta$ : $1.50\left(\mathrm{~s}, 6 \mathrm{H},-\left(\mathrm{CH}_{3}\right)_{2}\right), 3.06\left(\mathrm{~s}, 3 \mathrm{H}, 5-\mathrm{CH}_{3}\right), 3.59$ (s, $\left.2 \mathrm{H},-\mathrm{CH}_{2} \mathrm{OH}\right), 4.13\left(\mathrm{~s}, 3 \mathrm{H}, 6-\mathrm{CH}_{3}\right), 5.16(\mathrm{~s}, 1 \mathrm{H}$, $\mathrm{OH}), 7.27$ (m, 1H, 8-H), 7.57 (m, 2H, 9-H, 7-H), $8.15\left(\mathrm{~d}, J_{4-3}=6.1 \mathrm{~Hz}, 1 \mathrm{H}, 4-\mathrm{H}\right), 8.21\left(\mathrm{~d}, J_{10-8}=7.5\right.$ $\mathrm{Hz}, 1 \mathrm{H}, 10-\mathrm{H}), 8.39$ (d, $\left.J_{3-4}=6.0 \mathrm{~Hz}, 1 \mathrm{H}, 3-\mathrm{H}\right), 8.43$ (s, 1H, -CONH), 9.70 (s, 1H, 11-H).

9-hydroxymethyl-5,6-dimethyl-1-[N,N-(dimethylamino)ethyl]carbamoyl-6H-pyrido[4,3-b]carbazole (13a)

Yield: $44 \%$; mp: $131-132^{\circ} \mathrm{C}$. Analysis: calcd. for $\mathrm{C}_{23} \mathrm{H}_{26} \mathrm{~N}_{4} \mathrm{O}_{2}$ : C, 70.75; $\mathrm{H}, 6.71 ; \mathrm{N}, 14.35 \%$; found: $\mathrm{C}, 70.52 ; \mathrm{H}, 6.82 ; \mathrm{N}, 14.09 \% .{ }^{1} \mathrm{H}$ NMR $\left(\mathrm{DMSO}_{6}\right) \delta: 2.11\left(\mathrm{~m}, 2 \mathrm{H}, \beta-\mathrm{CH}_{2}\right), 2.24(\mathrm{~s}, 6 \mathrm{H}$, $\left.\mathrm{N}\left(\mathrm{CH}_{3}\right)_{2}\right), 3.11$ (s, 3H, 5- $\left.\mathrm{CH}_{3}\right), 3.50$ (q, 2H, $\alpha-\mathrm{CH}_{2}$ ), 4.13 (s, 3H, 6- $\left.\mathrm{CH}_{3}\right), 4.67$ (s, $\left.2 \mathrm{H},-\mathrm{CH}_{2} \mathrm{OH}\right), 5.28$ (s, $1 \mathrm{H},-\mathrm{OH}), 7.53(\mathrm{~m}, 2 \mathrm{H}, 8-\mathrm{H}, 7-\mathrm{H}), 8.16(\mathrm{~m}, 2 \mathrm{H}, 4-$ $\mathrm{H}, 10-\mathrm{H}), 8.42\left(\mathrm{~d}, J_{3-4}=6.0 \mathrm{~Hz}, 1 \mathrm{H}, 3-\mathrm{H}\right), 8.77(\mathrm{t}$, $\left.1 \mathrm{H},-\mathrm{CONHCH}_{2}\right), 9.66$ (s, 1H, 11- H).

9-hydroxymethyl-5,6-dimethyl-1-[N,N-(dimethylamino)propyl]carbamoyl-6H-pyrido[4,3-b]carbazole (13b)

Yield: $33 \%$; mp: $129-130^{\circ} \mathrm{C}$. Analysis: calcd. for $\mathrm{C}_{24} \mathrm{H}_{28} \mathrm{~N}_{4} \mathrm{O}_{2}$ : C, 71.26; H, 6.98; N, $13.85 \%$ found: $\mathrm{C}$, 71.11; H, 7.16; N, 13.58\%. ${ }^{1} \mathrm{H}$ NMR (DMSO-d $\left.{ }_{6}\right) \delta$ : $1.74\left(\mathrm{~m}, 2 \mathrm{H}, \beta-\mathrm{CH}_{2}\right), 2.19\left(\mathrm{~s}, 6 \mathrm{H},-\mathrm{N}\left(\mathrm{CH}_{3}\right)_{2}\right), 2.37$ (t, $\left.2 \mathrm{H}, \gamma-\mathrm{CH}_{2}\right), 3.07$ (s, 3H, 5- $\left.\mathrm{CH}_{3}\right), 3.39\left(\mathrm{~m}, 2 \mathrm{H}, \alpha-\mathrm{CH}_{2}\right)$, $4.15\left(\mathrm{~s}, 3 \mathrm{H}, 6-\mathrm{CH}_{3}\right), 4.67$ (s, 2H, 9- $\left.\mathrm{CH}_{2} \mathrm{OH}\right), 5.25$ (s, $1 \mathrm{H},-\mathrm{OH}), 7.54$ (m, 2H, 8-H, 7-H), 8.16 (m, 2H, 4-H, $10-\mathrm{H}), 8.42\left(\mathrm{~d}, J_{3-4}=6.0 \mathrm{~Hz}, 1 \mathrm{H}, 3-\mathrm{H}\right), 8.93(\mathrm{t}, J \mathrm{NH}-$ $\left.\mathrm{CH}_{2}=5.7 \mathrm{~Hz}, 1 \mathrm{H},-\mathrm{CONHCH}_{2}\right), 9.54(\mathrm{~s}, 1 \mathrm{H}, 11-\mathrm{H})$. 
9-hydroxymethyl-5,6-dimethyl-1-(2-hydroxy-1,1dimethylethyl)carbamoyl-6H-pyrido[4,3-b]carbazole (13c)

Yield: $37 \%$; mp: $205-206^{\circ} \mathrm{C}$. Analysis: calcd. for $\mathrm{C}_{23} \mathrm{H}_{25} \mathrm{~N}_{3} \mathrm{O}_{3}$ : C, 70.57; $\mathrm{H}, 6.44 ; \mathrm{N}, 10.73 \%$; found: $\mathrm{C}, 70.35 ; \mathrm{H}, 6.63 ; \mathrm{N}, 10.58 \% .{ }^{1} \mathrm{H}$ NMR $\left(\mathrm{DMSO}-\mathrm{d}_{6}\right) \delta: 1.44\left(\mathrm{~s}, 6 \mathrm{H},-\left(\mathrm{CH}_{3}\right)_{2}\right), 3.06(\mathrm{~s}, 3 \mathrm{H}$, $\left.5-\mathrm{CH}_{3}\right), 3.57\left(\mathrm{~s}, 2 \mathrm{H},-\mathrm{CH}_{2} \mathrm{OH}\right), 4.13\left(\mathrm{~s}, 3 \mathrm{H}, 6-\mathrm{CH}_{3}\right)$, $4.67\left(\mathrm{~s}, 2 \mathrm{H}, 9-\mathrm{CH}_{2} \mathrm{OH}\right), 5.16\left(\mathrm{~s}, 1 \mathrm{H},-\mathrm{CH}_{2} \mathrm{OH}\right), 5.27$ (s, 1H, 9- $\left.\mathrm{CH}_{2} \mathrm{OH}\right), 7.54(\mathrm{~m}, 2 \mathrm{H}, 8-\mathrm{H}, 7-\mathrm{H}), 8.16(\mathrm{~m}$, $2 \mathrm{H}, 4-\mathrm{H}, 10-\mathrm{H}), 8.39$ (d, $\left.J_{3-4}=6.0 \mathrm{~Hz}, 1 \mathrm{H}, 3-\mathrm{H}\right)$, 8.44 (s, 1H, -CONH), 9.73 (s, 1H, 11-H).

1-ethoxycarbonyl-5,6-dimethyl-6H-pyrido[4,3-b]carbazole (9)

A mixture of 1-ethoxycarbonyl-5-methyl- $6 \mathrm{H}$ pyrido[4,3- $b$ carbazole 5 ( $0.60 \mathrm{~g}, 2 \mathrm{mmol}), 1.0 \mathrm{~g}$ dry potassium carbonate, $30 \mathrm{~mL}$ dimethyl carbonate, $3 \mathrm{~mL}$ DMF and 0.02 g 18-crown-6 was stirred at boiling temperature for $24 \mathrm{~h}$. After evaporation under reduced pressure the resulting compound was solubilized in water $(100 \mathrm{~mL})$ and extracted with methylene chloride. The extract was dried over magnesium
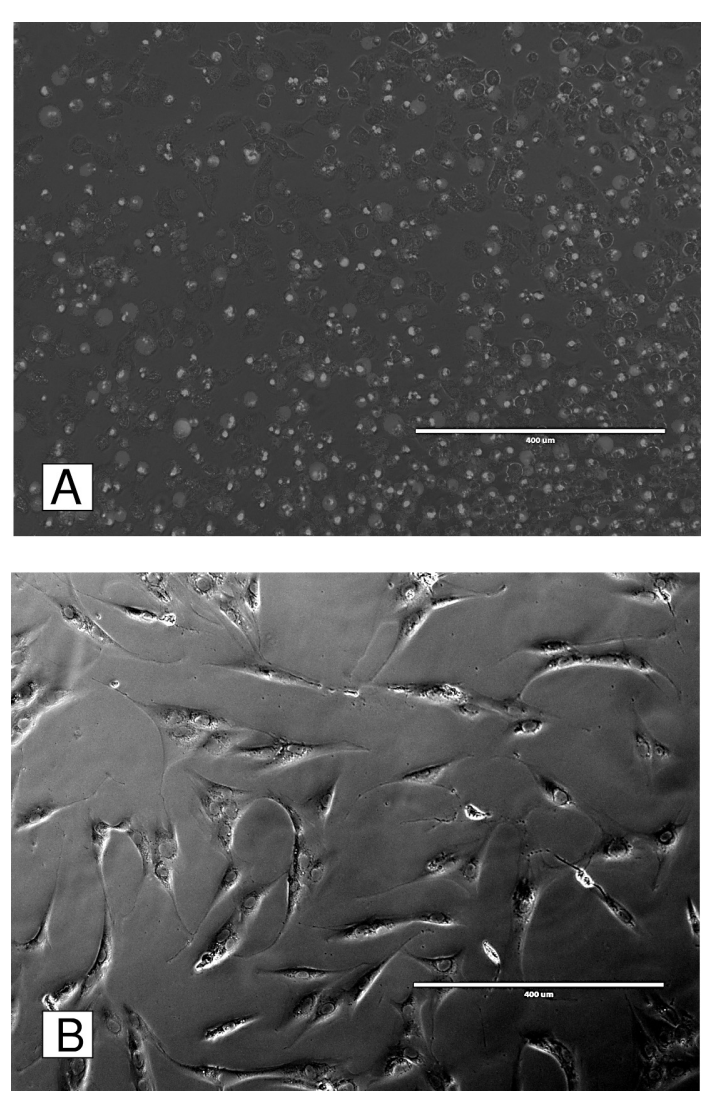

Figure 2. Cytotoxicity evaluation of compound $15(10 \mu \mathrm{M})$ for A549 (A) and NHDF (B) cells. Dyeing with propidium iodide (red cells - dead). Magnification of the 10 x lens, Ex: 531 nm Em: 593 $\mathrm{nm}$ sulfate. After evaporation of the solvent, the solid was purified using column chromatography and silica gel, eluting with methylene chloride: methanol, 99:1 v/v. Yield: $88 \% \mathrm{mp}: 191^{\circ} \mathrm{C}$. Analysis: calcd. for $\mathrm{C}_{20} \mathrm{H}_{18} \mathrm{~N}_{2} \mathrm{O}_{2}$ : C, 75.45; H, 5.70; N, 8.80\%; found: $\mathrm{C}$, 75.24; H, 5.89; N, 8.71\%. ${ }^{1} \mathrm{H}$ NMR (DMSO-d $\left.{ }_{6}\right) \delta$ : $1.42\left(\mathrm{t}, 3 \mathrm{H},-\mathrm{CH}_{2} \mathrm{CH}_{3}\right), 3.10\left(\mathrm{~s}, 3 \mathrm{H}, 5-\mathrm{CH}_{3}\right), 4.10$ (s, $\left.3 \mathrm{H}, 6-\mathrm{CH}_{3}\right), 4.56$ (q, $\left.2 \mathrm{H},-\mathrm{CH}_{2} \mathrm{CH}_{3}\right), 7.29(\mathrm{~m}, 1 \mathrm{H}$, $8-\mathrm{H}), 7.61(\mathrm{~m}, 2 \mathrm{H}, 7-\mathrm{H}, 9-\mathrm{H}), 8.22\left(\mathrm{~d}, J_{4-3}=6.3 \mathrm{~Hz}\right.$, $1 \mathrm{H}, 4-\mathrm{H}), 8.33$ (d, $\left.J_{10-8}=7.6 \mathrm{~Hz}, 1 \mathrm{H}, 10-\mathrm{H}\right), 8.45$ (d, $\left.J_{3-4}=6.3 \mathrm{~Hz}, 1 \mathrm{H}, 3-\mathrm{H}\right), 8.96(\mathrm{~s}, 1 \mathrm{H}, 11-\mathrm{H})$.

9-(3-carboxy-2,2-dimethylpropyl)acetate-5,6-dimethyl-1-[N,N-(dimethylamino)ethyl]carbamoyl6H-pyrido[4,3-b]carbazole (14)

3,3-Dimethylglutaric anhydride $0.14 \mathrm{~g}(1 \mathrm{mmol})$ was added to a mixture of 5,6-dimethyl-9-hydroxymethyl-1-[N,N-(dimethylamino)ethyl]carbamoyl-6H-pyrido[4,3- $b$ ]carbazole 13a in $20 \mathrm{~mL}$ of pyridine, anhydrous. The mixture was stirred at boiling temperature for $24 \mathrm{~h}$. After evaporation of the solvent, the solid was purified using column chromatography and silica gel, eluting with methylene chloride: methanol, 9 : $1 \mathrm{v} / \mathrm{v}$. Yield: $28 \% \mathrm{mp}$ : $107-$ $108^{\circ} \mathrm{C}$. Analysis: calcd. for $\mathrm{C}_{30} \mathrm{H}_{36} \mathrm{~N}_{4} \mathrm{O}_{5}: \mathrm{C}, 67.65 ; \mathrm{H}$, $6.81 ; \mathrm{N}, 10.52 \%$; found: $\mathrm{C}, 67.42 ; \mathrm{H}, 6.92 ; \mathrm{N}$, $10.33 \% .{ }^{1} \mathrm{H}$ NMR (DMSO-d $\left.)_{6}\right) \delta: 1.03$ (s, 6H, $\left.-\left(\mathrm{CH}_{3}\right)_{2}\right), 2.27\left(\mathrm{~s}, 2 \mathrm{H},-\mathrm{CH}_{2} \mathrm{COOCH}_{2}\right), 2.32(\mathrm{~s}, 6 \mathrm{H}$, -N $\left.\left(\mathrm{CH}_{3}\right)_{2}\right), 2.44\left(\mathrm{~s}, 2 \mathrm{H}, \mathrm{HOOCCH}_{2}\right), 2.77(\mathrm{~m}, 2 \mathrm{H}, \beta-$ $\mathrm{CH}_{2}$ ), 3.08 (s, 3H, 5- $\left.\mathrm{CH}_{3}\right), 3.49\left(\mathrm{~m}, 2 \mathrm{H}, \alpha-\mathrm{CH}_{2}\right)$, $4.16\left(\mathrm{~s}, 3 \mathrm{H}, 6-\mathrm{CH}_{3}\right), 5.25\left(\mathrm{~s}, 2 \mathrm{H}, 9-\mathrm{CH}_{2}\right), 7.61(\mathrm{~m}$, $2 \mathrm{H}, 8-\mathrm{H}, 7-\mathrm{H}), 8.19$ (d, 2H, $\left.J_{4-3}=6.1 \mathrm{~Hz}, 1 \mathrm{H}, 4-\mathrm{H}\right)$, 8.25 (s, 1H, 10-H), 8.44 (d, $\left.J_{3-4}=6.0 \mathrm{~Hz}, 1 \mathrm{H}, 3-\mathrm{H}\right)$, 8.44 (s, 1H, -CONH), 9.65 (s, 1H, 11-H).

5,6-dimethyl-1-[N,N-(dimethylamino)ethyl]carbamoyl-9-(N-methylcarbamoyloxymethyl)-6Hpyrido[4,3-b]carbazole (15)

A mixture of compound 13a $0.38 \mathrm{~g}(1 \mathrm{mmol})$, $50 \mathrm{~mL}$ dry chloroform and $0.36 \mathrm{~g}(3 \mathrm{mmol}) 4-$ dimethylaminopyridine (DMAP) was stirred at room temperature for $0.5 \mathrm{~h}$, following with addition of $5 \mathrm{~mL}$ methyl isocyanate at room temperature over $2 \mathrm{~h}$. The resulting mixture was solubilized in water $(100 \mathrm{~mL})$ and extracted with chloroform. The extract was dried over magnesium sulfate. After evaporation of the solvent, the solid was purified using column chromatography and silica gel, eluting with chloroform: methanol, $99: 1 \mathrm{v} / \mathrm{v}$.

Yield: $60 \% \mathrm{mp}: 185-186^{\circ} \mathrm{C}$. Analysis: calcd. for $\mathrm{C}_{25} \mathrm{H}_{29} \mathrm{~N}_{5} \mathrm{O}_{3}$ : C, 67.13; $\mathrm{H}, 6.48 ; \mathrm{N}, 15.65 \%$; found: $\mathrm{C}, 67.05 ; \mathrm{H}, 6.53 ; \mathrm{N}, 15.73 \% .{ }^{1} \mathrm{H} \mathrm{NMR}$ $\left(\mathrm{DMSO}_{6}\right) \delta=$ ppm: $2.25\left(\mathrm{~s}, 6 \mathrm{H}, 2 \mathrm{x} \mathrm{CH}_{3}\right), 2.53(\mathrm{~m}$, $\left.2 \mathrm{H}, \beta-\mathrm{CH}_{2}\right), 2.59$ (d, $\left.J=4.5 \mathrm{~Hz}, 3 \mathrm{H}, \mathrm{CH}_{3} \mathrm{NH}\right), 3.09$ 
(s, 3H, 5- $\left.\mathrm{CH}_{3}\right), 3.51\left(\mathrm{~m}, 2 \mathrm{H}, \alpha-\mathrm{CH}_{2}\right), 4.12(\mathrm{~s}, 3 \mathrm{H}, 6-$ $\left.\mathrm{CH}_{3}\right), 5.18\left(\mathrm{~s}, 2 \mathrm{H}, 9-\mathrm{CH}_{2}\right), 7.11(\mathrm{~d}, J=4.4 \mathrm{~Hz}, 1 \mathrm{H}$, $\left.\mathrm{CH}_{3} \mathrm{NH}\right), 7.65$ (s, 2H, 8-H+ 7-H), 8.15 (d, $J_{4-3}=6.1$ $\mathrm{Hz}, 1 \mathrm{H}, 4-\mathrm{H}), 8.19$ (s, 1H, 10-H), 8.42 (d, $J_{3-4}=6.1$ $\mathrm{Hz}, 1 \mathrm{H}, 3-\mathrm{H}), 8.78$ (t, $\left.J=5.5 \mathrm{~Hz}, 1 \mathrm{H}, \mathrm{CONH}-\mathrm{CH}_{2}\right)$, $9.66(\mathrm{~s}, 1 \mathrm{H}, 11-\mathrm{H})$.

\section{Biological tests}

Test solutions of nine new pyrido[4,3- $b]$ carbazole derivatives 8 a-b, 10 a-b, 13 a-c, 14, 15 and ellipticine $(1 \mathrm{mg} / \mathrm{mL})$ were prepared ex tempore for each test by dissolving each of the compounds in $100 \mu \mathrm{L}$ of DMSO and $900 \mu \mathrm{L}$ of culture media. The solutions were diluted in cell culture media to reach the final concentrations of 100 to $0.0001 \mu \mathrm{g} / \mathrm{mL}$. The compounds were weighed (Sartorius) with an accuracy of $0.00001 \mathrm{~g}$.

\section{Cell lines}

The human cancer cell lines A498 (kidney cancer) and A549 (non-small-cell lung cancer) were used. The lines were cultured by the Cell Culture Collection of the Department of Tumor Immunology, Institute of Immunology and Experimental Therapy, Wrocław, Poland. The normal cells (normal human dermal fibroblasts - NHDF) were purchased from LONZA (Verviers, Belgium).

The A549 cells were cultivated in the RPMI 1640 Opti- MEM medium supplemented with 5\% fetal calf serum (FCS), glutamine $(2 \mathrm{mM})$, penicillin (100 U/mL), and streptomycin $(100 \mu \mathrm{g} / \mathrm{mL})$. The A498 cells were cultivated in Eagle medium supplemented with $10 \%$ serum (FCS), sodium pyruvate $(1 \mathrm{mM})$, glutamine $(2 \mathrm{mM})$, penicillin $(100 \mathrm{U} / \mathrm{mL})$, and streptomycin $(100 \mu \mathrm{g} / \mathrm{mL})$. The NHDF cells were cultivated in DMEM medium supplemented with $10 \%$ fetal bovine serum (FBS), glutamine (2 $\mathrm{mM})$, penicillin $(100 \mathrm{U} / \mathrm{mL})$, and streptomycin $(100 \mu \mathrm{g} / \mathrm{mL})$. The cell cultures were maintained at $37^{\circ} \mathrm{C}$ in a humid atmosphere containing $5 \% \mathrm{CO}_{2}$.

\section{SRB}

The SRB method was used as described by Skehan et al. (21). The cytostatic assays were performed after 72-hour exposure of the cultured cells to varying concentrations of the tested agents. Each experiment was repeated three times. The effect of DMSO on the proliferation of selected cell lines was tested.

\section{DISCUSSION}

The nine newly synthesized compounds were subjected to the preliminary in vitro tests for their cytostatic activity against two human tumor cell lines
A498 (kidney cancer) and A549 (lung cancer) in comparison to reference compounds, ellipticine and doxorubicin (standard cytostatic). Pharmacological results were outlined in Table 1. All compounds exhibited biological activity. Compounds with substituents at 1 and 9 positions (13a, 13b, 13c, 14, 15) showed significant antiproliferative properties. The results of the in vitro biological study indicated that the presence of $[N, N$-(dimethylamino)ethyl $]$ carbamoyl moieties at 1 position of $6 H$-pyrido[4,3-b]carbazole ring system significantly increases the cytostatic properties of synthesized compounds when compared to $[N, N$-(dimethylamino)propyl $]$ carbamoyl moieties at 1 position of $6 H$-pyrido[4,3-b]carbazole. Compound 13a outperformed the antiproliferative action on both tumor cell lines tested when compared to ellipticine and doxorubicin. New pyridocarbazole derivative 15 (Polish patent PL-218501) showed significant antiproliferative properties comparable to reference ellipticine and doxorubicin on human tumor cell lines A498. The compounds $\mathbf{1 5}$ and 8a showed biological activity only slightly worse or comparable to reference ellipticine and doxorubicin on human tumor cell lines A549. All the tested compounds also showed lower toxicity towards normal cells (NHDF). High antiproliferative activity of the new derivatives in relation to cancer cells and low toxicity to normal cells are an important feature of the synthesized compounds (Fig. 2).

As it has been shown previously (3), application of structure-activity relationship (SAR) to design of new active compounds can result in the determination of the chemical group responsible for induction of a desired biological effect. In our case, SAR based approach produced derivatives that outperformed the reference compound in several tests. Further investigation of the reported compounds is in progress.

\section{Conflict of interest}

The authors declare that there is no conflict of interest.

\section{REFERENCES}

1. Hansch C.: Acc. Chem. Res. 2, 232 (1969).

2. Hansch C., Hoekman D., Leo A., Zhang L., Li P.: Toxicol. Lett. 45, 79 (1995).

3. Ferlin M., Marzano C., Gandin V., Dall'Acqua S., Dalla L.: ChemMedChem. 4, 363 (2009).

4. Jasztold-Howorko R., Landras C., Pierré A., Atassi G., Guilbaud N. et al.: Med. Chem. 37, 2445 (1994). 
5. Le Mée S., Chaminade F., Delaporte C., Markovits J., Saucier J.M., Jacquemin-Sablon A.: Mol. Pharmacol. 4, 58 (2000).

6. Guilbaud N., Kraus-Berthier L., Saint-Dizier D., Rouillon M.H., Jan M. et al.: Cancer Chemother. Pharmacol. 138, 513 (1996).

7. Kraus-Berthier L., Guilbaud N., Jan M., SaintDizier D., Rouillon M.H. et al.: Eur. J. Cancer. 33, 1881 (1997).

8. Mée S. Le., Pierré A., Markovits J., Atassi G., Jacquemin-Sablon A., Saucier J.M.: Mol. Pharmacol. 53, 213 (1998).

9. Pichard-Garci L., Weaver R.J., Eckett N., Scarfe G., Fabre J.M. et al.: Drug Metab. Dispos. 32, 80 (2004).

10. Giacchetti S., Cornez N., Eftekhari P., Awada A., Cuvier C. et al.: Proc. Am. Assoc. Cancer Res. 39, 324 (1998).

11. Malonne H., Farinelle S., Decaestecker C., Gordower L., Fontaine J. et al.: Clin. Cancer Res. 6, 3774 (2000).

12. Kraus-Berthier L., Guilbaud N., Léonce S., Parker T., Genissel P. et al.: Cancer Chemother. Pharmacol. 50, 95 (2002).
13. Awada A., Giacchetti S., Gerard B., Eftekhary P., Lucas C. et al.: Ann. Oncol. 13, 1925 (2002).

14. Pivot X., Awada A., Gedouin D., Kerger J., Rolland F. et al.: Ann. Oncol. 114, 373 (2003).

15. Vassal G., Merlin J.L., Terrier-Lacombe M.J., Grill J., Parker F. et al.: Cancer Chemother. Pharmacol. 51, 385 (2003.)

16. Tylińska B., Jasztold-Howorko R., Mastalarz H., Szczaurska-Nowak K., Wietrzyk J.: Arch. Pharm. 341, 351 (2008).

17. Tylińska B., Jasztold-Howorko R., Mastalarz H., Kłopotowska D., Filip B., Wietrzyk J.: Acta Pol. Pharm. 67, 495 (2010).

18. Tylińska B., Jasztold-Howorko R., Mastalarz H., Szczaurska-Nowak K., Materek P., Wietrzyk J.: Acta Pol. Pharm. 68, 31 (2011).

19. Jasztold-Howorko R., Tylińska B., Biaduń B., Gębarowski T., Gąsiorowski K.: Acta Pol. Pharm. 70, 823 (2013).

20. Besselièvre R., Husson H.P.: Tetrahedron 37, (Suppl. 1), 241 (1981).

21. Skehan P., Storeng R., Scudiero D., Monks A., Mcmahon J. et al.: J. Natl. Cancer Inst. 82, 1107 (1990).

Received: 21.12 .2017 